\title{
On large plasmoid formation in a global magnetohydrodynamic simulation
}

\author{
I. Honkonen ${ }^{1,2}$, M. Palmroth ${ }^{1}$, T. I. Pulkkinen ${ }^{1,{ }^{*}}$, P. Janhunen ${ }^{1}$, and A. Aikio ${ }^{3}$ \\ ${ }^{1}$ Finnish Meteorological Institute, Helsinki, Finland \\ ${ }^{2}$ Department of Physics, University of Helsinki, Helsinki, Finland \\ ${ }^{3}$ Department of Physical Sciences, University of Oulu, Oulu, Finland \\ * currently at: School of Electrical Engineering, Aalto University, Finland
}

Received: 25 May 2010 - Revised: 9 December 2010 - Accepted: 10 January 2011 - Published: 14 January 2011

\begin{abstract}
We investigate plasmoid formation in the magnetotail using the global magnetohydrodynamic (MHD) simulation GUMICS-4. Here a plasmoid implies a major reconfiguration of the magnetotail where a part of the tail plasma sheet is ejected downstream, in contrast to small Earthwardpropagating plasmoids. We define a plasmoid based solely on the structure of the closed (connected to the Earth at both ends) magnetic field line region. In this definition a plasmoid is partly separated from the ordinary closed field line region by lobe field lines or interplanetary field lines resulting from lobe reconnection. We simulate an event that occurred on 18 February 2004 during which four intensifications of the auroral electroject (AE) index occurred in $8 \mathrm{~h}$. Plasmoids form in the simulation for two of the four AE intensifications. Each plasmoid forms as a result of two consecutive large and fast rotations of the interplanetary magnetic field (IMF). In both cases the IMF rotates 180 degrees at 10 degrees per minute, first from southward to northward and some $15 \mathrm{~min}$ later from northward to southward. The other two AE intencifications however are not associated with a plasmoid formation. A plasmoid does not form if either the IMF rotation speed or the angular change of the rotation are small. We also present an operational definition for these fully connected plasmoids that enables their automatic detection in simulations. Finally, we show mappings of the plasmoid footpoints in the ionosphere, where they perturb the polar cap boundary in both hemispheres.
\end{abstract}

Keywords. Magnetospheric physics (Magnetospheric configuration and dynamics; Magnetotail)

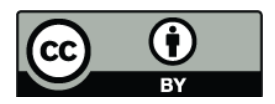

Correspondence to: I. Honkonen (ilja.honkonen@fmi.fi)

\section{Introduction}

The solar wind energy drives all dynamic phenomena within the near-Earth space. The basic process of extracting solar wind energy is called a substorm, during which solar wind energy is first loaded into the magnetosphere during the growth phase and subsequently released during the substorm expansion phase (McPherron, 1991). During the recovery phase, the magnetospheric and ionospheric dynamics subside. The growth phase starts typically after the interplanetary magnetic field (IMF) turns southward, and the dayside reconnection begins to bring more magnetic energy to the tail lobes. The new magnetic flux added to the tail lobes stretches the tail and compresses the plasma sheet, increasing the intensity of the duskward cross-tail current as well. At some point, the tail current disrupts, leading to two phenomena (McPherron, 1991): (1) the cross-tail current is forced to divert via the ionosphere and (2) a part of the tail is released downwind. These ejected large magnetic structures are called plasmoids. The onset mechanism of the current disruption and the chain of events prior to the onset are still unknown and under vigorous research. While Hsu and McPherron (2002) showed that half of all substorms are triggered by northward turning of the interplanetary magnetic field (IMF), half of the substorms are associated with no particular changes within the solar wind driver and may be driven by an internal plasma instability within the magnetotail (e.g. Coppi et al., 1966).

Plasmoids are large magnetic structures that form in the Earth's magnetotail and remove plasma and energy from the magnetosphere (Hones, 1979). During a plasmoid formation, the three-dimensional structure of the magnetotail becomes complicated, with spatially alternating closed and open magnetic topologies. Figure 1 shows the traditional two-dimensional description of a plasmoid as closed loops

Published by Copernicus Publications on behalf of the European Geosciences Union. 


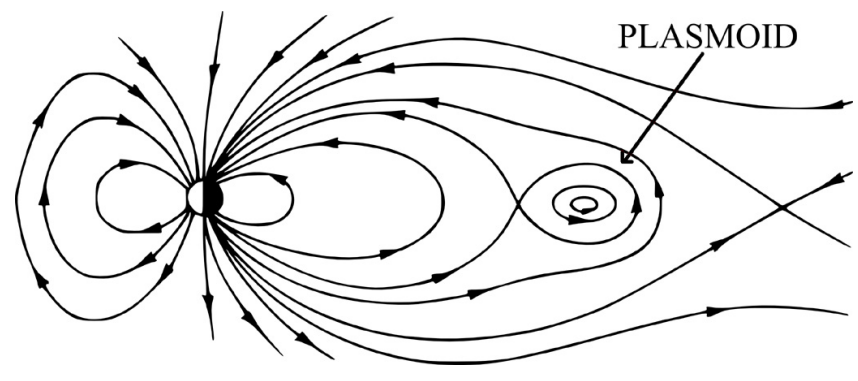

Fig. 1. A plasmoid forming inside the closed magnetic field line region in the $y$-plane. Original figure by D. P. Stern.

of magnetic field lines that are not connected to the Earth (original figure by D. P. Stern). The closed loops form when regions of the plasma sheet are severed from the Earth by magnetic reconnection in the near-Earth tail (Hones et al., 1984a). In three dimensions, with non-zero $B_{\mathrm{y}}$-component in the plasma sheet, reconnection creates a flux rope of closed field lines that remain connected to the Earth (Hughes and Sibeck, 1987). Each magnetic field line belongs to one of the following topologies: (1) closed field line with both ends in the ionosphere, (2) IMF field line with both ends in the solar wind, (3)-(4) lobe field line with one end in the ionosphere and the other in the solar wind, (5) a closed loop that is not attached to the Earth. If the symmetry $\left(B_{\mathrm{y}}=0\right)$ that leads to topology (5) is removed, plasmoid field lines belong to topologies 1...4, which often seems to be the case (Moldwin and Hughes, 1992). Figure 2 shows a sketch of this situation. Therefore, without $B_{\mathrm{y}}=0$ symmetry plasmoids in the general 3-D case cannot be defined by the type of the magnetic field lines alone. A plasmoid could be defined, for example, as magnetic field lines that cross the equatorial plane more than once. However, this definition is arbitrary as it depends on the chosen plane and the number of crossings (Birn et al., 1989).

The size of plasmoids has varied greatly in observations, e.g. in the x-direction from $4 \ldots 10 R_{\mathrm{E}}$ (Ieda et al., 1998) and $16.7 \pm 13.0 R_{\mathrm{E}}$ (Moldwin and Hughes, 1992) up to $75 \ldots 150 R_{\mathrm{E}}$ (Hones et al., 1984b), depending on their distance from the Earth. Estimations of the energy carried away by plasmoids have also varied by an order of magnitude, from about $0.2 \times 10^{15} \mathrm{~J}$ (Ieda et al., 1998) to $4 \times 10^{15} \mathrm{~J}$ (Silbergleit et al., 1997). In these studies plasmoids were detected mainly from the magnetic field and plasma data of ISEE-3 or Geotail spacecraft. The large error estimates were due to the fact that data was only available from a single spacecraft.

Although it is well established that plasmoids are mostly associated with substorms (Slavin et al., 1987; Moldwin and Hughes, 1993), typically it is only stated that plasmoids form due to reconnection of closed magnetic field lines in the near-

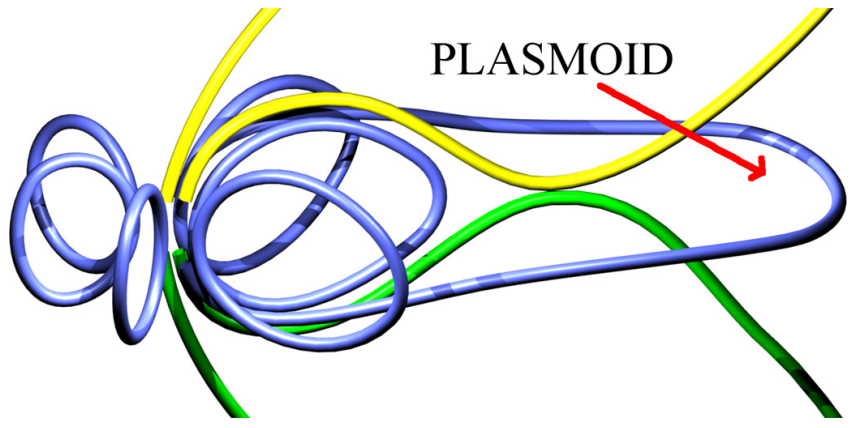

Fig. 2. A plasmoid consisting of the closed magnetic field line region partially detached from the Earth. Closed magnetic field lines are colored blue. Magnetic field lines attached to the Earth only in the parallel and anti-parallel directions (lobe field lines) are colored yellow and green, respectively.

Earth tail (Hones, 1977, 1979; Hones et al., 1984b; Birn et al., 1989; Moldwin and Hughes, 1992; Slinker et al., 1995; Ieda at al., 2001; Farr et al., 2008). In this paper we examine the details of plasmoid formation using simulation results for the event of 18 February 2004, from the global magnetohydrodynamic simulator GUMICS-4 (Janhunen, 1996). We define a plasmoid as a major reconfiguration of the magnetotail where part of the tail plasma sheet is ejected downstream, in contrast to Earthward-propagating small plasmoids (see for example Zong et al., 2004). First we describe the event of 18 February 2004, the delay from ACE to the magnetopause and compare simulation results with Cluster observations. Then we present a method for identifying tail plasmoids in simulations based on the structure of the closed magnetic field lines, e.g. connected plasmoids, and show the large-scale evolution of the magnetotail in the simulation. Finally, we show the effects of the plasmoid footpoints in the simulation ionosphere and discuss plasmoid formation in light of observations.

\section{Event description and the solar wind driver}

Figure 3 illustrates the calculation of the delay from ACE spacecraft (located at GSE $\left.(221,-22,9) R_{\mathrm{E}}\right)$ to the magnetopause. The delay is computed by correlating upstream ACE measurements with those of GOES-12 and Cluster 1. Upstream measurements are delayed $0 . .4000 \mathrm{~s}$ in $10 \mathrm{~s}$ increments and a linear least squeares value is calculated for all delays. GOES-12 was near local noon at the time of arrival of a solar wind pressure pulse. The pulse, detected at Earth at 16:40 UT, caused an increase of the dayside magnetic field in the magnetosphere (Fig. 3a). The linear least squares value is minimized with a delay of $2720 \mathrm{~s}$, about $45 \mathrm{~min}$.

Cluster 1 traversed the dayside magnetosheath during the period 19:30 to 22:30 UT. During that period Cluster $1 \mathrm{ob}-$ served several sign changes in $B_{\mathrm{z}}$, which are correlated to 
ACE measurements delayed to the magnetopause
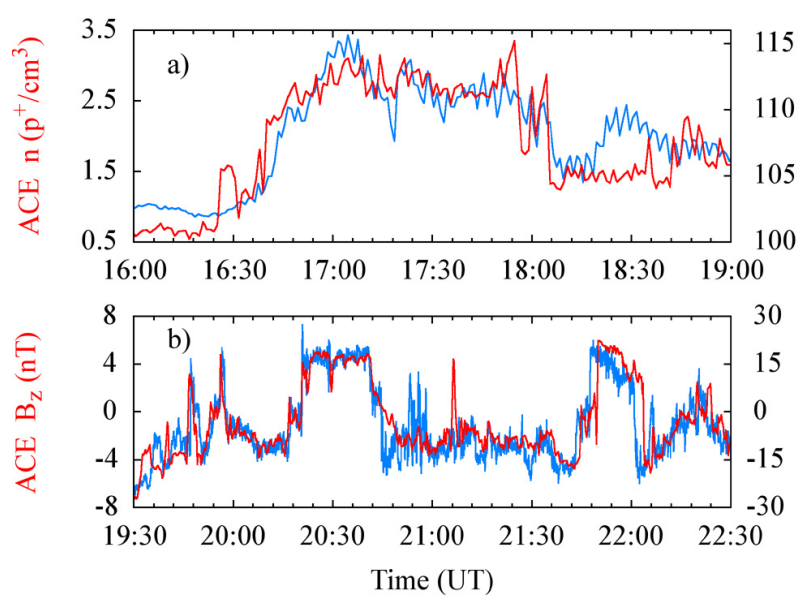

Fig. 3. The solar wind $n$ and $B_{Z}$ from ACE delayed by about 45 and 46 min over $n$ from GOES-12 and $B_{\mathrm{Z}}$ from Cluster 1, respectively. Note that the panels are displayed at different times.

ACE measurements. The linear least squares value between ACE and Cluster $1 B_{\mathrm{Z}}$ is minimized using a delay of $2770 \mathrm{~s}$, about $46 \mathrm{~min}$ (Fig. 3b). The average of the two estimates ( $2745 \mathrm{~s}$ or $46 \mathrm{~min}$ ) is used as the delay to the magnetopause in the following analysis. Figure $3 \mathrm{~b}$ shows that the delayed $\mathrm{ACE} B_{\mathrm{Z}}$ measurements precede those of Cluster 1 before 20:00 UT and lag behind Cluster 1 measurements after 21:30 UT. This implies an increase in the solar wind bulk speed during that time, which indeed is the general trend (see Fig. 4).

Figure 4 shows the solar wind data from ACE delayed to the magnetopause using the $46 \mathrm{~min}$ delay. The solar wind density (Fig. 4a) fluctuated between 1 and $3 \mathrm{~cm}^{-3}$ during the event with large jumps recorded at 16:40 and 19:45 UT. The solar wind bulk speed (Fig. 4b) increased from $440 \mathrm{~km} \mathrm{~s}^{-1}$ to $500 \mathrm{~km} \mathrm{~s}^{-1}$ at $16: 40 \mathrm{UT}$ and fluctuated between 460 and $500 \mathrm{~km} \mathrm{~s}^{-1}$ for the rest of the day. The most striking features in the solar wind parameters during this event were changes in the IMF. Its Z-component (Fig. 4c) changed sign more than a dozen times, varying between -8 and $8 \mathrm{nT}$. The IMF clock angle (Fig. 4d) varied between -140 and $20^{\circ}$ several times during the event. The clock angle is defined by the direction of the IMF in the GSE yz-plane as $0^{\circ}$ in the positive $\mathrm{z}$ direction and ranging from $-180^{\circ}$ to $180^{\circ}$. The $\mathrm{X}$-component of IMF did not show large features except for a sinusoidal change around 18:30 UT.

The provisional AE index on 18 February 2004 presented in Fig. $4 \mathrm{~g}$ shows four intensifications between 16:00 and 24:00 UT. The first AE intensification at 16:00 UT does not seem to be directly driven by the solar wind, as all parameters are fairly constant during that time. The increse (growth phase) of the second AE intensification starts as the IMF turns southward at 18:50. This is followed by the sudden in-
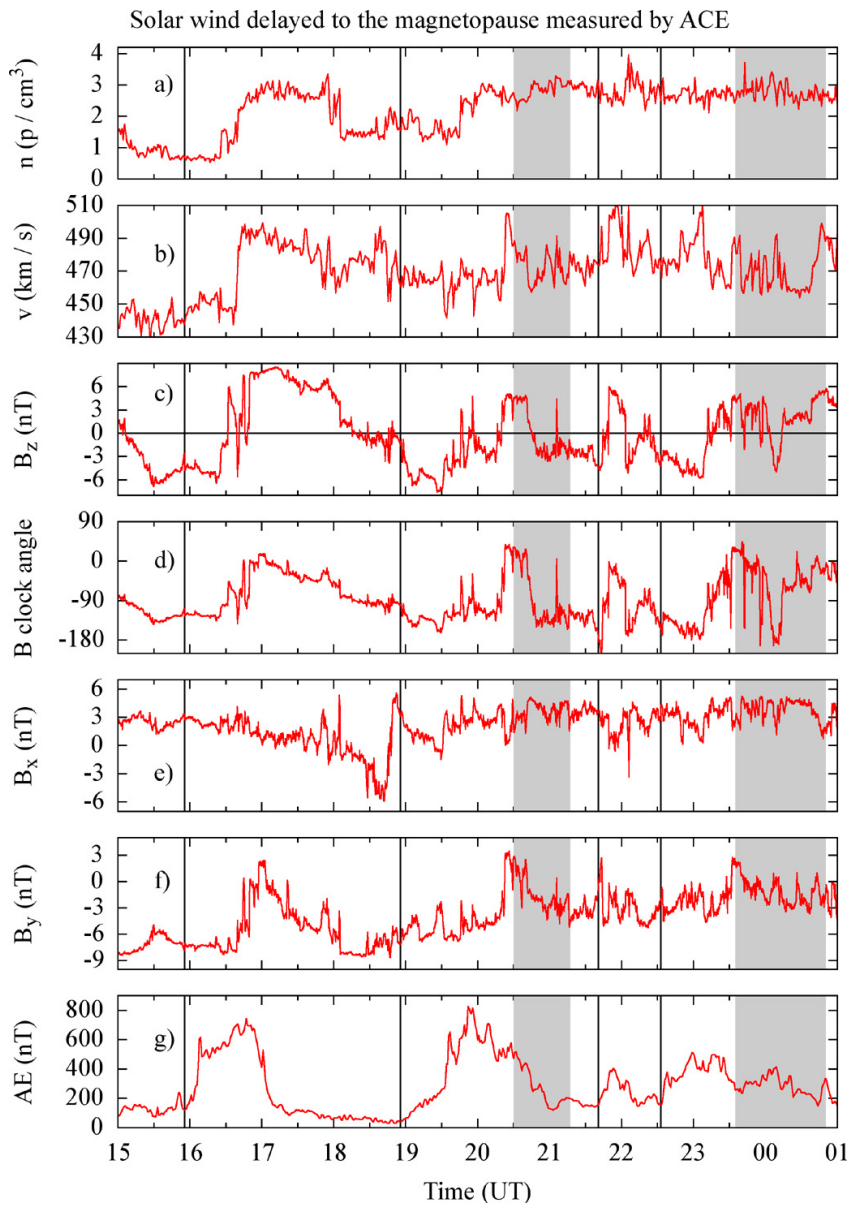

Fig. 4. (a) density, (b) velocity, (c) $B_{\mathrm{Z}}$, (d) clock angle, (e) $B_{\mathrm{X}}$, (f) $B_{\mathrm{y}}$ of the delayed solar wind measured by ACE on 18 February 2004 at $x_{\mathrm{GSE}}=221 R_{\mathrm{E}}$ and $(\mathrm{g})$ the provisional AE index from $\mathrm{Ky}-$ oto $\mathrm{AE}$ index service. Vertical black lines mark the start of the AE intensifications, vertical grey bars mark the plasmoids formed in the simulation.

tensification of AE (substorm expansion phase) about $45 \mathrm{~min}$ later when $B_{\mathrm{z}}$ suddenly jumps from -7 to $-3 \mathrm{nT}$. The recovery phase starts around 20:30 after the IMF has turned northward.

\section{Simulation}

\subsection{GUMICS-4}

The GUMICS-4 global magnetosphere-ionosphere coupling simulation (Janhunen, 1996, and references therein) solves the ideal MHD equations in fully conservative form. The simulation is robust and has been tested extensively against observations (e.g. Palmroth et al., 2003). Elliptic cleaning is used to enforce $\nabla \cdot \boldsymbol{B}=0$ (Brackbill and Barnes, 1980). GUMICS-4 uses a hierarchical cubic grid and also temporal subcycling which reduces the required computation time 


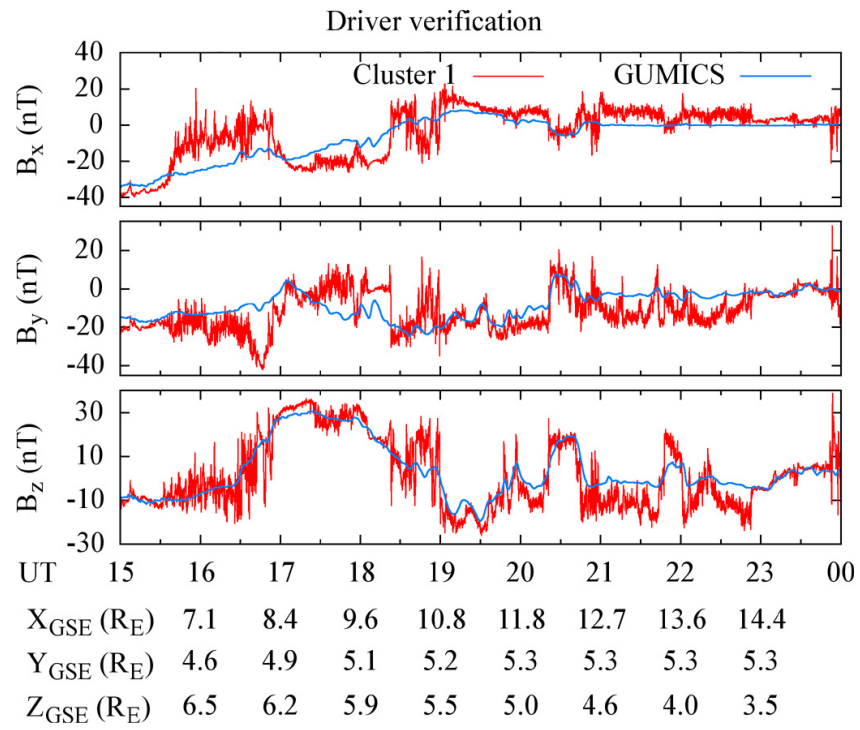

Fig. 5. The magnetic field from Cluster 1 and the simulation at the same location as function of time.

by at least an order of magnitude when compared to global timestepping. The simulation grid extends from $32 R_{\mathrm{E}}$ upstream to $224 R_{\mathrm{E}}$ downstream from the Earth and is $128 R_{\mathrm{E}}$ in the $y_{\mathrm{GSE}}$ and $z_{\mathrm{GSE}}$ directions. The simulation grid uses automatic cell refinement and the grid is adapted at run time based on local gradients, spatial coordinates and userspecified priority functions (Janhunen et al., 1996). The finest spatial resolution in the simulation used in this study is $1 / 4 R_{\mathrm{E}}$. GUMICS-4 takes as input the solar wind density, temperature, velocity and magnetic field at the upstream boundary and outputs the plasma parameters in the simulation box.

The inner boundary of the MHD simulation at $3.7 R_{\mathrm{E}}$ is coupled to the ionospheric solver. The electrostatic ionosphere model is coupled to the magnetosphere through fieldaligned currents and electron precipitation. The ionospheric electron density is solved in a 3-D grid with 20 non-uniform altitude levels, taking into account the magnetospheric fieldaligned current, source plasma density and temperature (Janhunen, 1996). Height-integrated Hall and Pedersen conductivities are obtained from the electron density and are used along with the field aligned current to solve the horizontal current distribution in the ionosphere. This gives the ionospheric potential which is used as the electric field in the MHD equations.

The GUMICS simulation was run using the solar wind observations of ACE from 14:00 to 24:00 UT on 18 February 2004. The IMF x-component was set to a constant value of $0 \mathrm{nT}$ in order to keep the magnetic field at the upstream boundary of the simulation divergence free. The dipole tilt angle was set at the start of the simulation and kept at a value of $-4.3^{\circ}$ for the entire run.

\subsection{Driver verification}

Figure 5 shows the simulation results along Cluster 1 orbit together with the measurements during the event. The observed $B_{\mathrm{Z}}$ component measured by the FGM instrument (Balogh et al., 2001) is well reproduced by GUMICS, as are some of the dynamics of the $B_{\mathrm{y}}$ component. The general trend in $B_{\mathrm{x}}$ is also reproduced by GUMICS. The observations of Geotail at $(-22.2 \ldots-21.4,-10.8 \ldots-18.2,5.3 \ldots 5.3) R_{\mathrm{E}}$ and Wind at $(-132.6 \ldots-130.0,-6.8 \ldots-8.6,11.6 \ldots 11.7) R_{\mathrm{E}}$ (both in GSE) are also reproduced by GUMICS quite well (Palmroth et al., 2010). On the whole the effects of the solar wind driver are reproduced in the simulation, giving credibility to the modelling results presented in this paper. However the key point here is to demonstrate that the solar wind driver measured at L1 far upstream of the Earth is indeed the same that impacted the magnetosphere 45 min later.

\section{Plasmoids in global MHD}

\subsection{Definition}

Plasmoid development can be divided into three distinct stages (Hughes and Sibeck, 1987): (1) reconnection within the closed magnetic field line region in the plasma sheet with $B_{\mathrm{y}} \neq 0$ creates helical field lines that are connected to the Earth along the flanks. This structure is known as a flux rope. A plasmoid is formed at this stage if it is defined, for example, as field lines that cross the equatorial plane more than once. (2) As the reconnection in (1) proceeds to lobe field lines the flux rope becomes enveloped in IMF field lines and also lobe field lines (Birn et al., 1989) in the central tail, and the flux rope starts to move tailward. At this stage the plasmoid is still connected to the Earth at both ends and can be identified from the topology of closed field lines alone, using a method which we will present next. The plasmoid is formed at this stage at the latest. (3) At some point the plasmoid will start to dissipate due to reconnection near the flanks between the plasmoid closed field lines and lobe field lines. Using the definition we present below, the plasmoid no longer exists at this stage (it is not connected to the Earth at both ends), but its remnants on IMF and lobe field lines can still be identified (Birn et al. 1989; Farr et al. 2008). In this work we will refer to flux ropes in stage 2), e.g. connected to the Earth at both ends, as plasmoids.

We define a plasmoid using the magnetic field topology, as a region of closed field lines detached from the quasidipolar tail region. Normally, closed magnetic field lines are bounded by a surface which encloses the Earth and the inner magnetosphere along quasi-dipolar field lines. Topologically, the surface has genus zero (e.g. a sphere). Reconnection within the plasma sheet breaks the surface, creating a hole in the closed field line region on the tailside (e.g. sphere with a handle attached). Topologically, the surface attains 


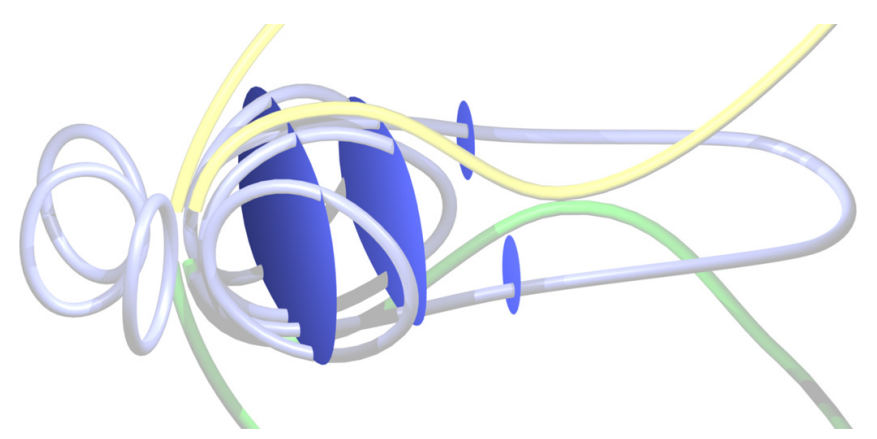

Fig. 6. The areas of closed magnetic field lines in three different x-planes added into Fig. 2.

genus one instead of the original genus zero. The space within that extra handle is called a plasmoid. It is possible for even multiple plasmoids to form (topological genus two or larger), but such structures did not form in the simulation of this event. Strictly speaking also a single plasmoid can have a topological genus larger than one: When a plasmoid has partially dissipated (Fig. 9f) the number of handles can be said to have increased although no additional plasmoids have formed.

The plasmoid search from the simulation results was conducted in the following way: Every cell in the simulation is classified by tracing the magnetic field line from the cell centroid. Four different topologies are possible: (1) closed, with both ends of the magnetic field line attached to the ionosphere, (2) open, with neither end attached to the ionosphere and (3)-(4) lobe field line, with one end attached to the ionosphere and the other not. After classification of the magnetic topology, the closed field line cells are identified at yz-planes starting from the plane $x=-10 R_{\mathrm{E}}$. A cluster of closed field line cells in a tail cross section is defined as a group of cells that share a vertex directly or through other cells in the same group (closed field lines cells). This is illustrated in Fig. 6 for three planes at different values of $x$. We define a plasmoid to begin at that value of $x$, where two or more such clusters also share a vertex through other closed field line cells downstream of that plane. In other words, a plasmoid is a singly connected 3-D set of closed field line cells, the 2-D intersection of which with some x-plane is multiply connected. Furthermore we define that a plasmoid constitutes the closed field line cells from the two or more clusters above and all other closed field line cells downstream that share a vertex directly with the clusters or through other closed field lines cells.

Although our definition of a plasmoid is different from Birn et al. (1989) and Farr et al. (2008), it does produce similar results. Our definition does not include free parameters and consequently the plasmoid search is straightforward to automate.

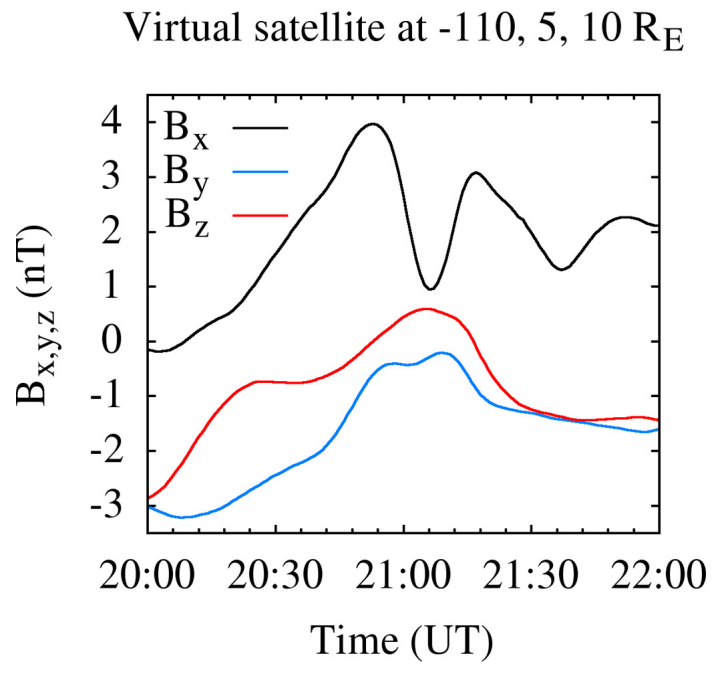

Fig. 7. The magnetic field observed in the simulation by a virtual satellite located at $(-110,5,10) R_{\mathrm{E}}$ in GSE during and after the second AE intencification.

\subsection{Plasmoid formation}

The simulated event consisted of four intensifications of the $\mathrm{AE}$ index (starting times marked with vertical black lines in Fig. 4). During that period the IMF $B_{\mathrm{y}}$ and $B_{\mathrm{z}}$ underwent several rapid changes. The solar wind density increased by at least a factor of 2 during the first two AE intensifications. In the simulation a plasmoid forms after the 2nd and 4th $\mathrm{AE}$ intensifications. The vertical grey bars in Fig. 4 show the time period during which the closed magnetic field line region starts to extend further downstream and when the plasmoid finally dissipates. Figure 7 shows the bipolar $B_{\mathrm{Z}}$ signature of the first plasmoid which formed around 21:00 UT as observed by a virtual satellite located at $(-110,5,10) R_{\mathrm{E}}$ in GSE. The plasmoid signature between 20:30 and 21:30 UT in the simulation agrees with the one used by Moldwin and Hughes (1992): a bipolar signature in $B_{\mathrm{y}}$ and/or $B_{\mathrm{Z}}$ not coincident with a neutral sheet crossing. Due to the small spatial resolution of the simulation in the tail the bipolar signature amplitude does not reach $3 \mathrm{nT}$.

Figures 8 to 10 show the magnetic topology classification in the simulation with the same color coding as in Fig. 2, with cells on a closed magnetic field line in blue and cells on a southern lobe field line in green. For clarity the northern lobe and IMF field line regions are not shown. Animation 1 shows the magnetic topology in the simulation with one minute intervals.

Figure 8 shows the magnetic field topology in the simulation during the first $\mathrm{AE}$ intensification at 16:31, 17:02, 17:21, 17:26, 18:02 and 18:25 UT. The IMF clock angle rotated from about $-130^{\circ}$ at $16: 40 \mathrm{UT}$ to $10^{\circ}$ at 17:00 UT and then returned slowly back to $-130^{\circ}$ between 17:00 and 19:00 UT. Due to dayside reconnection, the most sunward lobe field line 


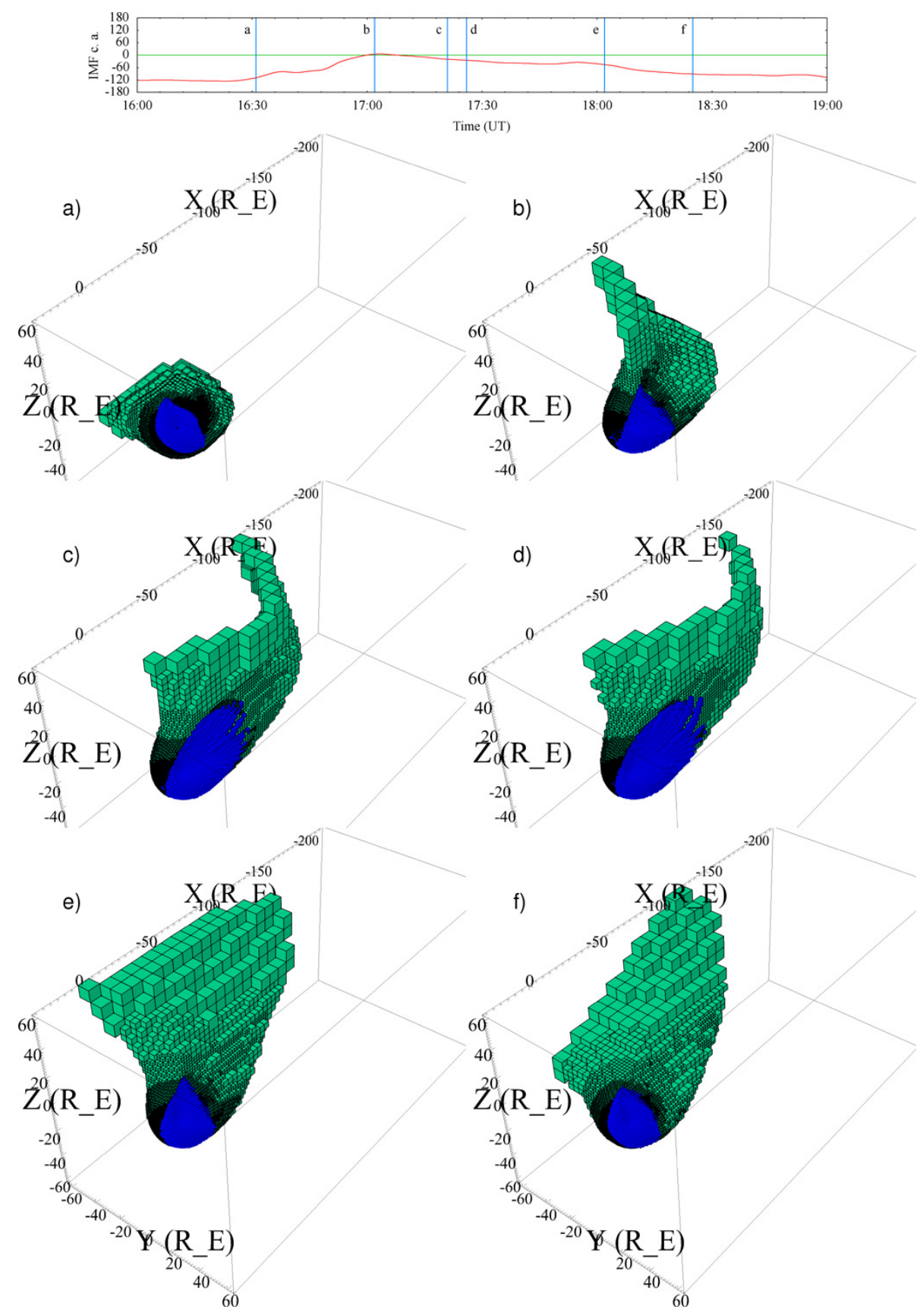

Fig. 8. Classification of cells in the simulation by their magnetic field topology is shown color coded. Blue cells show the region of closed magnetic field lines. Green cells show the region of southern lobe field lines. Northern lobe and IMF field line regions are not shown. The magnetic field topology in the simulation is shown during and after the first AE intencification at (a) 16:31, (b) 17:02, (c) 17:21, (d) 17:26, (e) 18:02 and (f) 18:25 UT. The IMF clock angle in each of the sub-panels is marked by blue bars at the top of the figure.

regions follow the direction of the IMF clock angle. This can be seen for southern lobe field lines in Figs. 8...10 as a green "wing" of lobe field lines on the dayside. In the case of positive IMF $B_{\mathrm{Z}}$, the southern lobe field lines drape over the closed field line region of the dayside magnetosphere towards the Northern Hemisphere, as seen at 17:02 and 17:21 UT in Fig. 8b-c. Figure 8 shows that changing the IMF clock angle increases the surface area of the lobe field line region perpendicular to the direction of solar wind bulk flow (yz-plane). The IMF rotation speed also affects how perpendicular the surface of the lobe field line region is to the solar wind bulk flow: faster rotation allows less time for the field lines to convect tailward thus producing a more perpendicular surface. At the same time the closed field 

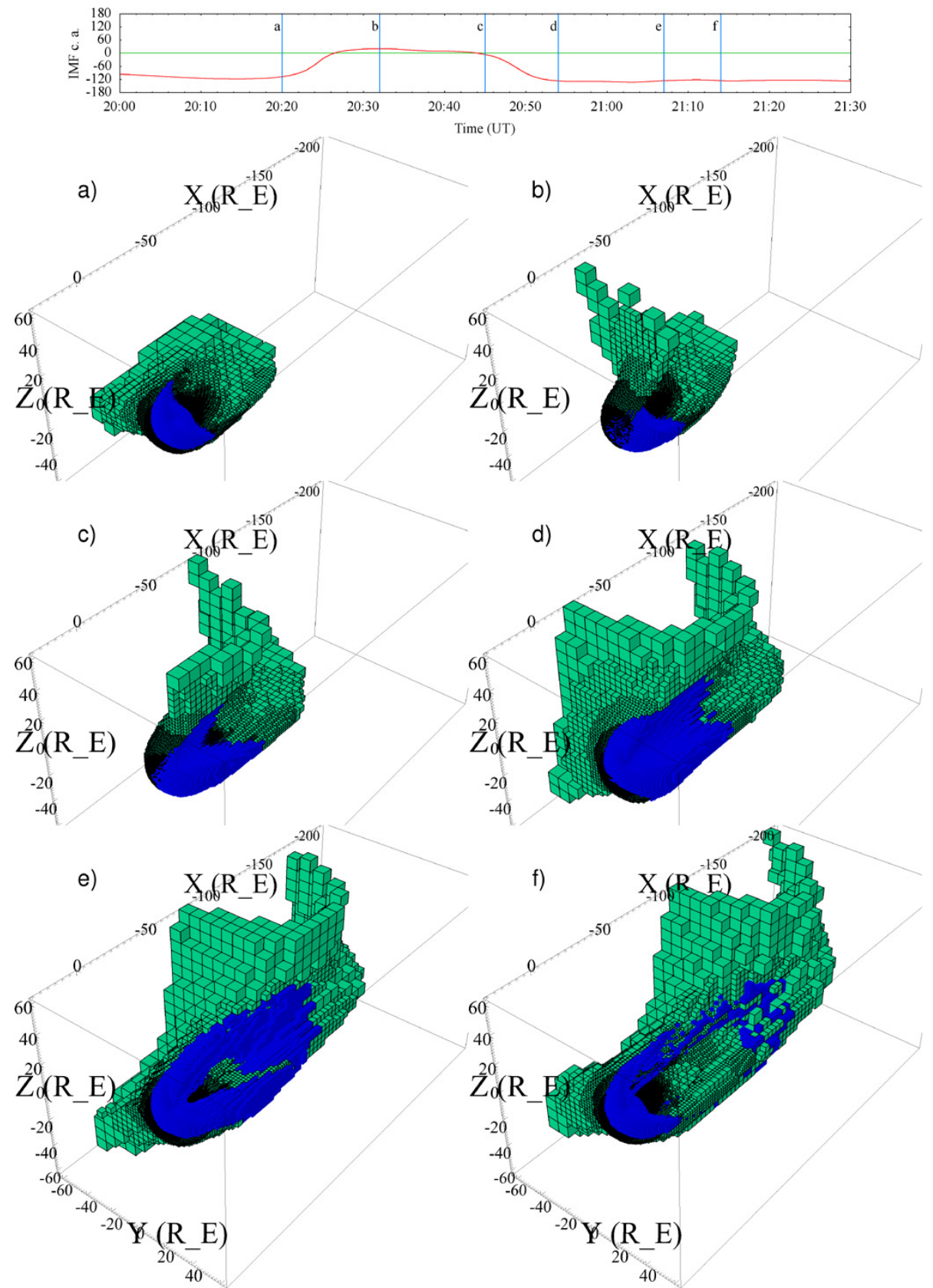

Fig. 9. Classification of cells in the simulation by their magnetic field topology is shown in the same format as in Fig. 8. The magnetic field topology and clock angle in the simulation are shown during and after the second AE intencification at (a) 20:20, (b) 20:32, (c) 20:45, (d) 20:54, (e) 21:07 and (f) 21:14 UT.

line region on the nightside of Earth extends downstream to about $50 R_{\mathrm{E}}$. Later (18:25 UT shown in Fig. 8f) the IMF clock angle slowly returns back to $-130^{\circ}$ between 17:00 and 19:00 UT and the closed field line region retreats back to about $20 R_{\mathrm{E}}$ downstream. A common feature in global simulations using ideal MHD is that the reconnection line forms quite close to the Earth at about $-20 R_{\mathrm{E}}$.

During the second AE intensification around 20:00 UT the IMF clock angle rotates rapidly from about $-120^{\circ}$ at 20:15 UT to $30^{\circ}$ at $20: 22 \mathrm{UT}$ and 20 min later back to $-130^{\circ}$ between 20:38 and 20:45 UT. This IMF rotation can also be seen in Fig. 9, where the most sunward southern lobe field lines again follow the IMF clock angle. The format of Fig. 9 is the same as for Fig. 8 and shows six snapshots from the simulation at 20:20, 20:32, 20:45, 20:54, 21:07 and 21:14 UT. This AE intensification differs from the first one mainly in the IMF rotation speed, particularly because the IMF also returns to its original direction in less than $15 \mathrm{~min}$. The average rotation speed during the second AE intensification is about $15^{\circ}$ per minute. The fast and large rotations 

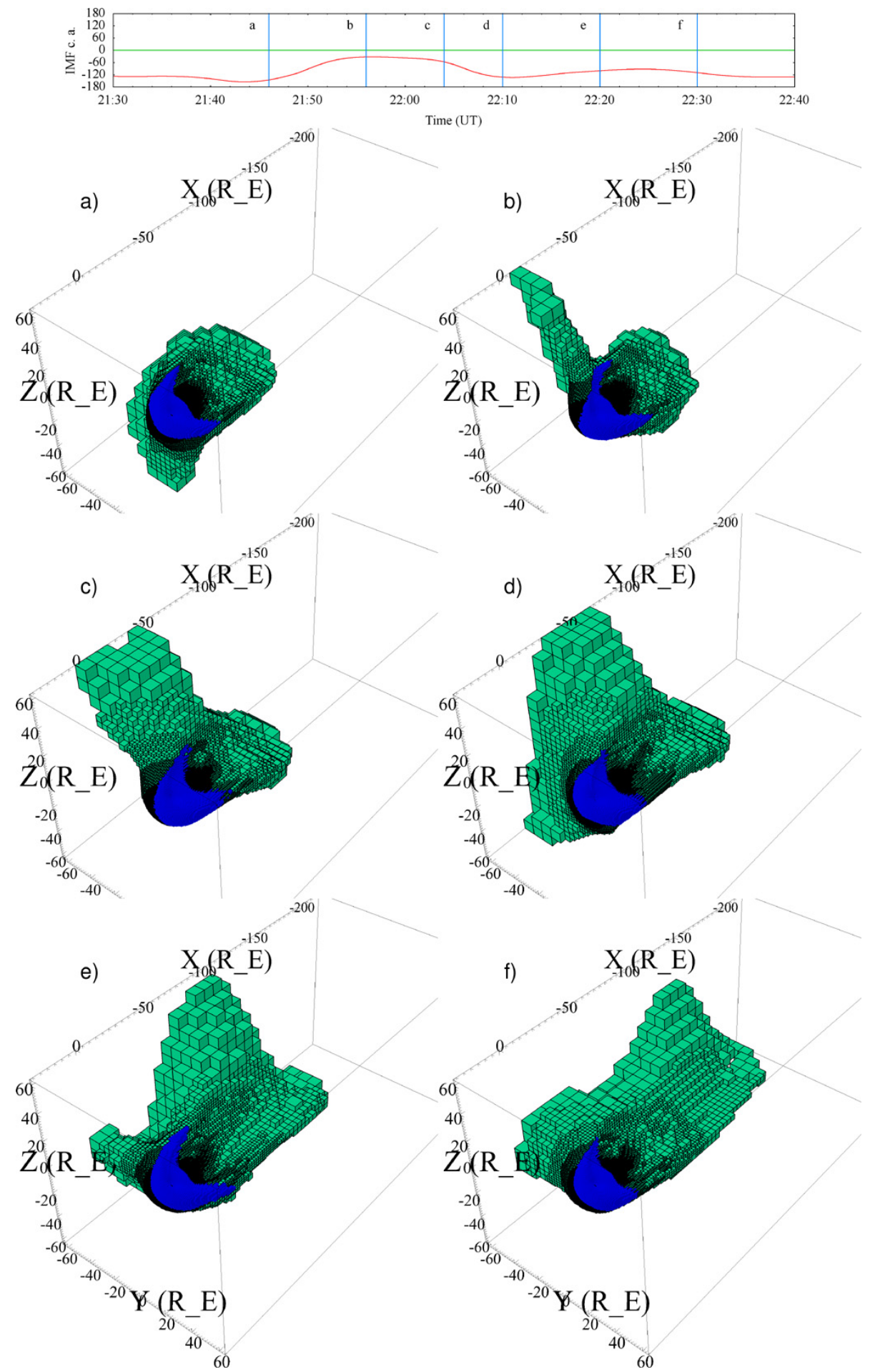

Fig. 10. Classification of cells in the simulation by their magnetic field topology is shown in the same format as in Fig. 8 . The magnetic field topology and clock angle in the simulation are shown during and after the third AE intencification at (a) 21:46, (b) 21:56, (c) 22:04, (d) 22:10, (e) 22:20 and (f) 22:30 UT.

of the IMF clock angle during the second AE intensification create large surfaces of lobe field lines almost perpendicular to the direction of the solar wind bulk flow. These surfaces also move downstream with the solar wind similarly to the first AE intensification in Fig. 8. Figure 9c shows that during the initial clock-wise IMF rotation the closed field line 

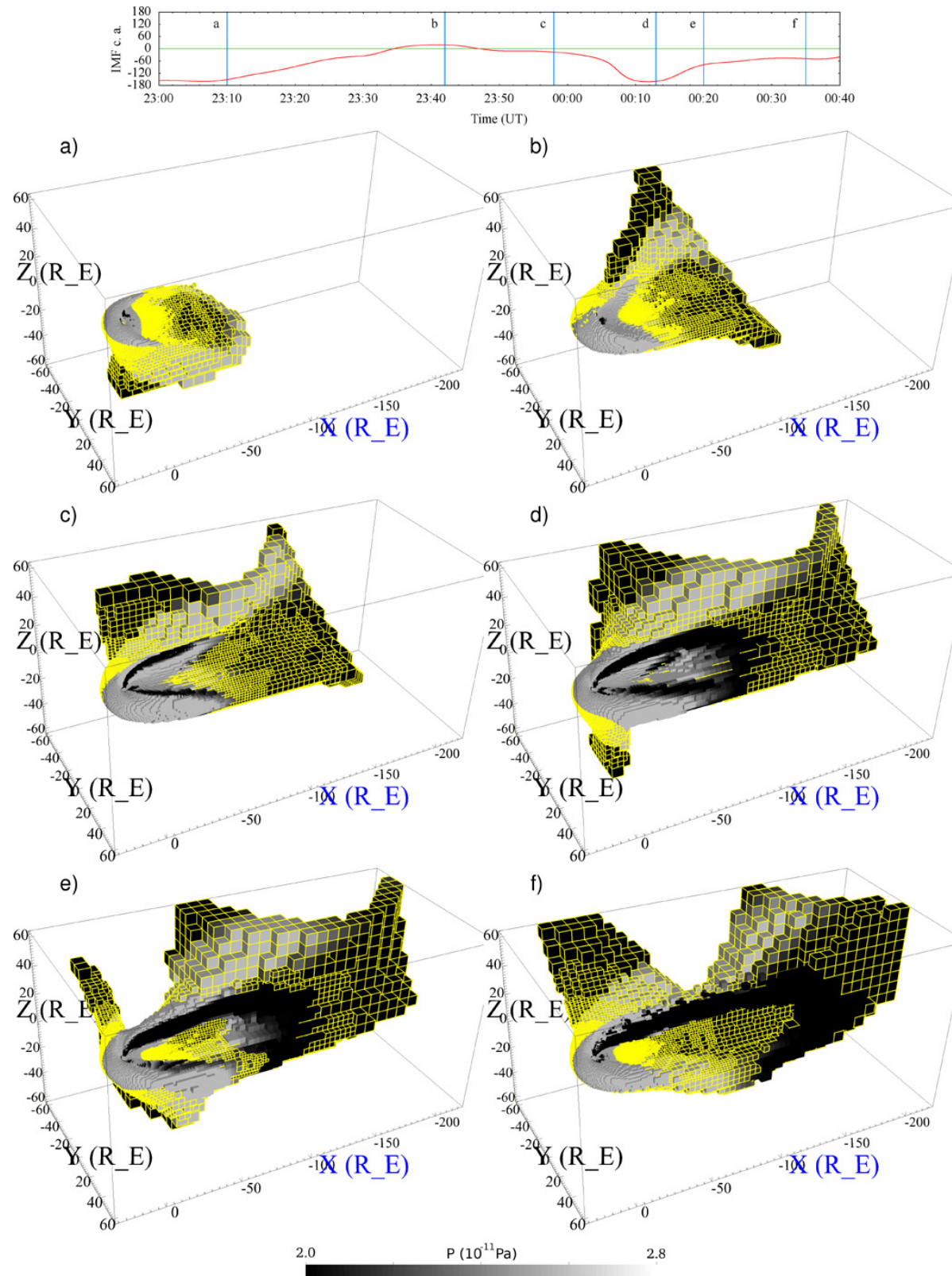

Fig. 11. Classification of cells in the simulation by their magnetic field topology is shown by the visible grid as in Fig. 8 . Color coding shows the thermal pressure. The magnetic field topology, thermal pressure and clock angle in the simulation are shown during and after the fourth AE intencification at (a) 23:10, (b) 23:42, (c) 23:58, (d) 00:13, (e) 00:20 and (f) 00:35 UT.

region does not extend as far downstream as during the first $\mathrm{AE}$ intensification (Fig. 8c). In contrast, when the IMF returns quickly to its original direction during the second $\mathrm{AE}$ intensification, the closed field line region extends even further downstream (Fig. 9d). As the lobe field line region is transported downstream, the closed field line region finally detaches from the nightside of the Earth (Fig. 9e) forming a plasmoid. The plasmoid starts to form at 20:30 UT during the recovery phase of the substorm. The space between the Earth and the plasmoid, for example in Fig. 9e, is not topologi- cally empty, but contains also northern lobe and IMF field lines which are not shown in the plot for clarity. In Fig. 9f the plasmoid has extended to about $-150 R_{\mathrm{E}}$ and has started to dissipate. Both Geotail and Wind are outside of the tail plasma sheet and do not show large plasmoid signatures during this event.

The third AE intensification around 22:00 UT differs from the previous two $\mathrm{AE}$ intensification in that the rotations of the IMF clock angle are smaller, only about $120^{\circ}$. The IMF rotation speed was of the same order as in the second 
$\mathrm{AE}$ intensification, about $20^{\circ}$ per minnute before and after 22:00 UT. Figure 10 shows the field line topologies during the third AE intensification at 21:46, 21:56, 22:04, 22:10, 22:20 and 22:30 UT in the same format as in Figs. 8 and 9. The dayside edge of the lobe field line region again follows the IMF clock angle. Figure 10 illustrates that a smaller rotation of the IMF creates a smaller surface of lobe field lines perpendicular to the solar wind bulk flow.

In other words, here the size of the surface perpendicular to solar wind bulk flow of lobe field lines depends only on the magnitude of the rotation of the IMF clock angle. This can be seen when comparing for example Figs. $9 \mathrm{~d}$ and $10 \mathrm{~d}$. In Fig. 9d the perpendicular lobe field line surface spans nearly $180^{\circ}$, but in Fig. 10d it is noticeably smaller. Also the second jump in the IMF clock angle during the third AE intensification seen in Fig. 10e and $\mathrm{f}$ seems to smooth out the perpendicular surface even further. The closed field line region does not seem to expand downstream during the third $\mathrm{AE}$ intensification.

The last AE intensification of the period was at 23:00 UT, and its IMF characteristics mostly resembled those during the second AE intensification: Namely, the IMF clock angle rotated $190^{\circ}$ between 23:07 and 23:30 UT with a speed of $8^{\circ}$ per minute and returned only $15 \mathrm{~min}$ later to its original direction of $-170^{\circ}$ between 23:55 and 00:15 UT. Figure 11 shows the simulation at 23:10, 23:42, 23:58, 00:13, 00:20 and 00:35 UT. Color coding shows the thermal pressure in each cell. The topology of the magnetic field is classified as in previous figures. The region of southern lobe field lines is indicated by the grid colored yellow and the closed field line region is shown without the grid. A second plasmoid forms in the simulation during the last AE intensification. As previously, the most dayside lobe field lines follow the IMF clock angle. During the fourth AE intensification, the topology of the magnetic field evolves similarly to the second AE intensification. First the IMF rotates quickly from $-170^{\circ}$ at 23:07 UT to $20^{\circ}$ at 20:30 UT and after about 15 min returns even faster to its original direction. Due to both rotations a large area of lobe magnetic field line is formed perpendicular to the solar wind bulk flow. Similarly to the second AE intensification, the lobe field line region moves downstream with the solar wind. The closed field line region on the night side also extends further downstream. As in Fig. 9 the region between the plasmoid and the Earth is not topologically empty, but contains also northern lobe and IMF field lines. Figure $11 \mathrm{~d}$ shows the closed field line region just before it detaches from the night side of the Earth at 00:13 UT and Fig. 11e shows the formed plasmoid $7 \mathrm{~min}$ later. The color coding in Fig. 11f and e shows that the thermal pressure in the upstream part of the plasmoid is larger than in the downstream part, indicating that the plasmoid is pushed downstream by pressure gradients.

\section{Plasmoid footpoints in the ionosphere}

Next we investigate the ionospheric mapping of the plasmoid. First we find the plasmoid in the simulation as desribed in Sect. 4.1. Then the magnetic field is traced from every cell of the plasmoid into the inner boundary of the simulation. Figure 12 shows the plasmoid footpoints in the "ionosphere" at $4 R_{\mathrm{E}}$ in red, other closed magnetic field lines in blue and northern lobe field lines in yellow. The same timesteps as in Fig. 9 viewed from about the same direction are shown in Fig. 12: 20:20, 20:32, 20:45, 20:54, 21:07 and 21:14 UT. Note how the rotating IMF distorts the polar cap boundary (Fig. 12c-d). Near the plasmoid footpoints the polar cap boundary shape is irregular (Fig. 12e). The ends of the plasmoid field lines map to the duskside of the Southern Hemisphere, where the shape of the polar cap boundary is also irregular (not shown). When the plasmoid has partially dissipated (Fig. 9f) the polar cap boundary in the simulation has returned to its original spherical shape as shown in Fig. 12f.

\section{Discussion}

In this paper we have presented GUMICS-4 simulation results of plasmoid formation during 18 February 2004. A plasmoid was defined as a major reconfiguration of the magnetotail where part of the tail plasma sheet is ejected downstream, in contrast to Earthward-propagating small plasmoids (see for example Zong et al., 2004). The event consists of four AE intensifications that occurred between 16:00 and 24:00 UT. During the event, GUMICS-4 reproduces in situ spacecraft observations quite well, indicating that the simulation results can be interpreted in light of physical processes in the near Earth space. We defined a plasmoid based only on the structure of the closed (connected to the Earth at both ends) magnetic field line region. We also presented an operational definition for these fully connected plasmoids that enable their automatic detection in simulations. The simulation results show the formation and detachment of a plasmoid after the second and fourth $\mathrm{AE}$ intensifications, while the other two AE intensifications were not associated with plasmoids. The plasmoid footpoints in the ionosphere form an irregularly shaped open-closed field line boundary.

Plasmoid formation is associated with sufficiently large and fast rotations of the IMF clock angle. This does not include such changes where one component of IMF is constant, e.g. northward turning of the IMF with constant $B_{\mathrm{y}}$. The IMF rotation creates large surfaces of lobe field lines perpendicular to the solar wind flow, which also drape over the closed field line region of the dayside magnetosphere due to lobe reconnection (Figs. 9 and 11). As the lobe field line region outside of the magnetosphere moves downstream with the solar wind, the closed field line region inside of the magnetosphere extends along the flanks, perhaps due to a viscous interaction 

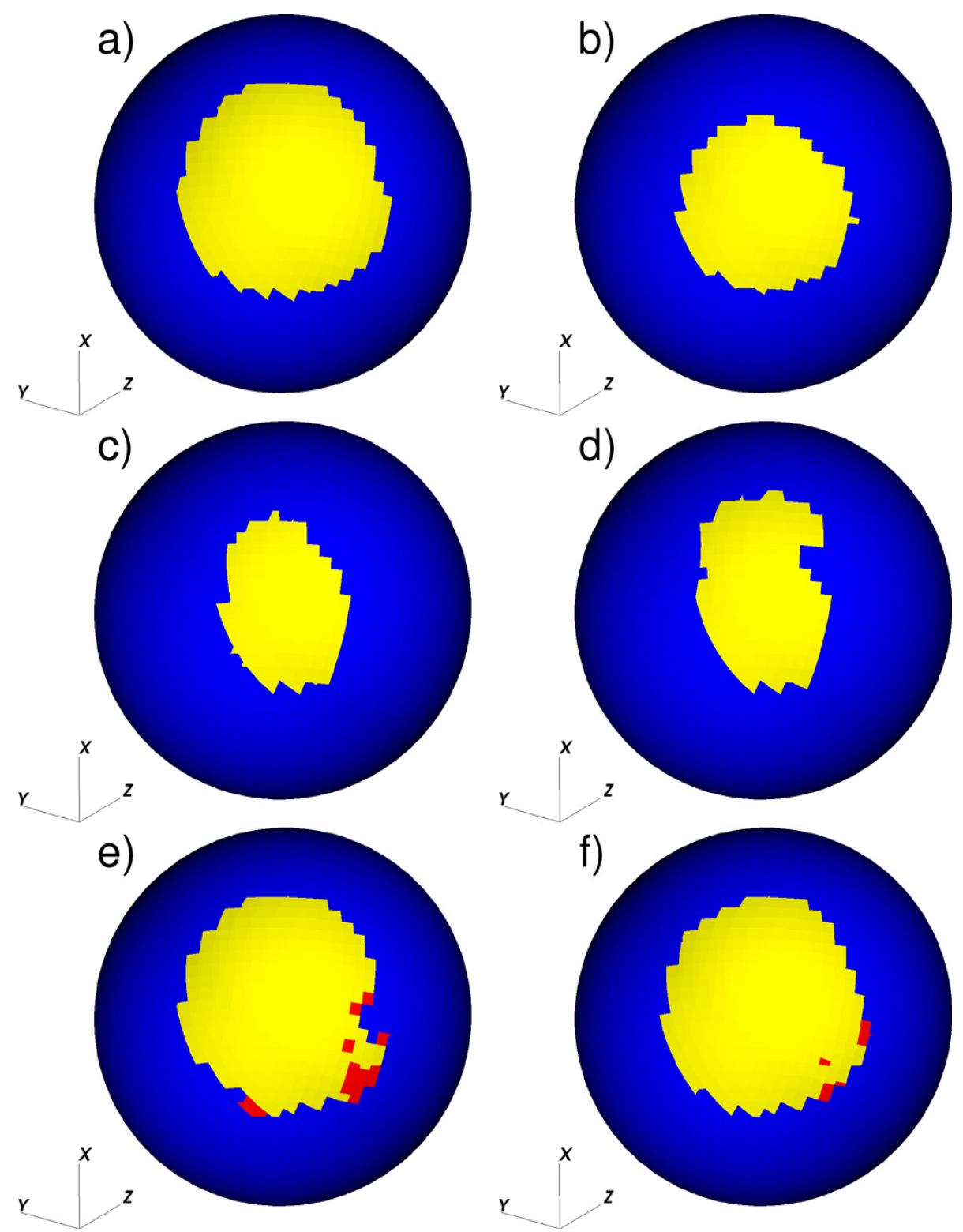

Fig. 12. The plasmoid footpoints at $4 R_{\mathrm{E}}$ in the Northern Hemisphere in the simulation are shown in red, other closed magnetic field lines in blue and northern lobe field lines in yellow. The same timesteps are shown as in Fig. 9: (a) 20:20, (b) 20:32, (c) 20:45, (d) 20:54, (e) 21:07 and (f) 21:14 UT.

between the lobe and closed field lines. The lobe field lines also pass through the tail center directly downstream of the Earth (at $y=0$ and $z=0$ ) and, as a result of magnetic tension (due to the frozen-in condition between magnetic field and plasma), move downstream also in that region. Consequently the closed field line region also expands downstream from the Earth to fill the void left by the lobe field line region. By this mechanism the closed field line region eventually expands beyond $x=-100 R_{\mathrm{E}}$ downstream and reconnection around $x=-50 R_{\mathrm{E}}$ creates the plasmoid. Plasmoid formation seems to be initiated during lobe reconnection after a fast northward rotation of the IMF. The substorm onset for the second $\mathrm{AE}$ intensification coincides with an IMF $B_{\mathrm{Z}}$ jump from -7 to $-3 \mathrm{nT}$ at 19:30 UT. In the simulation the large changes in IMF $B_{\mathrm{Z}}$ between 19:30 and 20:00 UT are smeared out and lobe reconnection starts only when during the large rotation of the IMF around 20:20 UT.

The literature lists several plasmoid features that are consistent with the results reported here: Hones et al. (1984b) reported on plasmoids of sizes from 75 to $150 R_{\mathrm{E}}$. Moldwin and Hughes (1992) surveyed 366 plasmoidlike structures and the events most comparable to our results are the isolated plasmoids with sizes up to $80 R_{\mathrm{E}}$. The larger of the two plasmoids in the GUMICS-4 simulation of this event is 
about $70 R_{\mathrm{E}}$ in the x-direction. Furthermore, Moldwin and Hughes (1992) observed that the size and speed of plasmoids as a function of distance downtail does not change beyond $100 R_{\mathrm{E}}$. This is in agreement with the simulation results, as the size and speed of the plasmoids were roughly constant after formation near $x_{\mathrm{GSE}}=-50 R_{\mathrm{E}}$ until their dissipation. The speed of the plasmoids reported here are close to the solar wind velocity. The observations of Moldwin and Hughes (1992) also suggest that plasmoids are open magnetic structures, possibly flux ropes, and not closed loops of magnetic field lines. In our simulation, the plasmoids form tailward of the reconnection line, while keeping some field lines attached to the Earth until dissipation. Nagai et al. (1997) concluded that there are two sites preferable for magnetic reconnection: inside $x_{\mathrm{GSM}}=-30 R_{\mathrm{E}}$ and near $x_{\mathrm{GSM}}=-100 R_{\mathrm{E}}$. In our simulation plasmoids are formed by reconnection under suitable IMF conditions around $x_{\mathrm{GSE}}=-50 R_{\mathrm{E}}$ (Figs. 9c and $\mathrm{d}, 11 \mathrm{c}$ and $\mathrm{d}$ ). After formation the plasmoids propagate downstream and are dissipated by reconnection near $x_{\mathrm{GSE}}=$ $-100 R_{\mathrm{E}}$ (Fig. 9f) or beyond $x_{\mathrm{GSE}}=-150 R_{\mathrm{E}}$ (Fig. 11f).

Pulkkinen et al. (1998) examined two sequential substorm onsets, of which the first one occurred during persistently negative IMF $B_{\mathrm{Z}}$ while the second one was associated with a northward turning of $B_{\mathrm{Z}}$. They observed that while the first onset remained localized, the second onset led to a major reconfiguration of the magnetotail. These observations are qualitatively in agreement with our simulation, which shows that a fast and large rotation of the IMF is required for plasmoid formation and launch, thus leading to a reconfiguration of the tail. Furthermore, according to Ieda at al. (2001), plasmoid formation is associated with the IMF first turning southward and then about $20 \mathrm{~min}$ later returning to northward. Here we report that the simulation plasmoids are formed when the delay between IMF rotations is from about 20 to $40 \mathrm{~min}$.

Kivelson et al. (1996) performed global MHD simulations using artificial solar wind as the simulation input, and showed that for a negative IMF $B_{\mathrm{y}}$ the flux rope closed field lines link the northern morning $(y<0)$ ionosphere to the southern evening $(y>0)$ ionosphere. This is also the case in our simulation, where IMF $B_{\mathrm{y}}$ is negative for at least an hour before the plasmoids form. In another global MHD simulation, Slinker et al. (1995) observed the formation of a plasmoid by southward turning of the IMF after a long period of steady northward IMF. Similarly to the results presented here, the formation region of plasmoids was at about $x=-45 R_{\mathrm{E}}$ and the plasmoid velocity approached the solar wind velocity.

Farr et al. (2008) presented LFM global MHD simulation (Lyon et al., 2004) results for a pair of substorms on $11 \mathrm{Au}-$ gust 2002. A flux tube formed in the simulation during southward $B_{\mathrm{Z}}$, following a north-south rotation about half an hour earlier. They mapped the flux tube into the ionosphere, and similarly to Kivelson et al. (1996), found that for positive IMF $B_{\mathrm{y}}$ the flux rope linked the northern dusk ionosphere to the southern dawn ionosphere. Furthermore, the ionospheric mapping showed a non-trivial structure of the flux rope inside of the closed field line region, in agreement with the results presented here.

The results presented here can also explain in part the close association observed between substorms and plasmoids (Moldwin and Hughes, 1993). Hsu and McPherron (2002) showed that half of all substorms are triggered by northward turning of the IMF. They did not, however, investigate simultaneous change in $B_{\mathrm{y}}$ during northward turning, which could have resulted in large rotation of the IMF instead. Based on our results this rotation could also create a plasmoid, especially if the IMF subsequently rotates again. Thus in some cases neither a substorm nor a plasmoid would result from the other, but instead they would be both the result of a fast rotation or rotations of the IMF clock angle.

Our main result in this paper is that the formation of plasmoids in the simulation is the result of two consecutive, sufficiently large and fast rotations of the IMF clock angle. As a result of these rotations the lobe field line region moving with the solar wind reduces the thermal pressure at the downstream edge of the closed field line region directly behind the Earth. Consequently the closed field line region extends downstream due to this pressure gradient, initiating the formation of a plasmoid. On the other hand when either the rotation speed of the IMF clock angle or the angle of rotation are small, the resulting pressure gradient is much smaller or nonexistent and a plasmoid does not form.

\section{Supplementary material related to this article is available online at: http://www.ann-geophys.net/29/167/2011/ angeo-29-167-2011-supplement.zip.}

Acknowledgements. This work is a part of the project 200141QuESpace, funded by the European Research Council under the European Community's seventh framework programme. The work of IH and MP is supported by project 218165 of the Academy of Finland. We thank the Cluster Active Archive, the FGM team and the instrument PI E. A. Lucek for providing freely the Cluster data. We also thank the Coordinated Data Analysis Web, the instrument PI N. Ness for providing the ACE data and the instrument PI H. Singer for providing the GOES data used in this study. Finally we thank the World Data Center for Geomagnetism for the Kyoto AE index service.

Topical Editor R. Nakamura thanks J. Birn and another anonymous referee for their help in evaluating this paper.

\section{References}

Balogh, A., Carr, C. M., Acuña, M. H., Dunlop, M. W., Beek, T. J., Brown, P., Fornacon, K.-H., Georgescu, E., Glassmeier, K.H., Harris, J., Musmann, G., Oddy, T., and Schwingenschuh, K.: The Cluster Magnetic Field Investigation: overview of in-flight 
performance and initial results, Ann. Geophys., 19, 1207-1217, doi:10.5194/angeo-19-1207-2001, 2001.

Birn, J., Hesse, M., and Schindler, K.: Filamentary Structure of a Three-Dimensional Plasmoid, J. Geophys. Res., 94, 241-251, 1989.

Brackbill, J. U. and Barnes, D. C.: The Effect of Nonzero $\nabla \cdot \mathbf{B}$ on the Numerical Solution of the Magnetohydrodynamic Equations, J. Comput. Phys., 35, 426-430, 1980.

Coppi, B., Laval, G., and Pellat, R.: Dynamics of the Geomagnetic Tail, Phys. Rev. Lett., 16, 1207-1210, 1966.

Farr, N. L., Baker, D. N., and Wiltberger, M.: Complexities of a 3-D plasmoid flux rope as shown by an MHD simulation, J. Geophys. Res., 113, A12202, doi:10.1029/2008JA013328, 2008.

Hones Jr., E. W.: Substorm Processes in the Magnetotail: Comments on "On Hot Tenuous Plasmas, Fireballs, and Boundary Layers in the Earth's Magnetotail” by L. A. Frank, K. L. Ackerson, and R. P. Lepping, J. Geophys. Res., 82, 5633-5640, 1977.

Hones Jr., E. W.: Transient phenomena in the magnetotail and their relation to substorms, Space Sci. Rev., 23, 393-410, 1979.

Hones Jr., E. W., Baker, D. N., Bame, S. J., Feldman, W. C., Gosling, J. T., McComas, D. J., Zwickl, R. D., Slavin, J. A., Smith, E. J., and Tsurutani, B. T.: Structure of the magnetotail at $220 R_{\mathrm{E}}$ and its response to geomagnetic activity, Geophys. Res. Lett., 11, 5-7, 1984.

Hones Jr, E. W., Birn, J., Baker, D. N., Bame, S. J., Feldman, W. C., McComas, D. J., Zwickl, R. D., Slavin, J. A., Smith, E. J., and Tsurutani, B. T.: Detailed examination of a plasmoid in the distant magnetotail with ISEE 3, Geophys. Res. Lett., 11, 10461049, 1984.

Hughes, W. J. and Sibeck, D. G.: On the 3-dimensional structure of plasmoids, Geophys. Res. Lett., 14, 636-639, 1987.

Hsu, T.-S. and McPherron, R. L.: An evaluation of the statistical significance of the association between northward turnings of the interplanetary magnetic field and substorm expansion onsets, J. Geophys. Res., 107, A11, doi:10.1029/2000JA000125, 2002.

Ieda, A., Machida, S., Mukai, T., Saito, Y., Yamamoto, T., Nishida, A., Terasawa, T., and Kokubun. S.: Statistical analysis of the plasmoid evolution with Geotail observations, J. Geophys. Res., 103, 4453-4465, 1998.

Ieda, A., Fairfield, D. H., Mukai, T., Saito, Y., Kokubun, S., Liou, K., Meng, C.-I., Parks, G. K., and Brittnacher, M. J.: Plasmoid ejection and auroral brightenings, J. Geophys. Res., 106, 38453857, 2001.

Janhunen, P.: GUMICS-3 - a global ionosphere-magnetosphere coupling simulation with high ionospheric resolution, Proceedings of Environmental Modelling for Space-Based Applications, 18-20 September 1996, Eur. Space Agency Spec. Publ., ESA SP-392, available at: http://www.space.fmi.fi/ pjanhune/papers/ ESTEC96/ESTEC96.ps.gz, 1996.

Janhunen, P., Koskinen, H. E. J., and Pulkkinen, T. I.: A new global ionosphere-magnetosphere coupling simulation utilizing locally varying time step, Proceeding of Third International Conference on Substorms, Versailles, France, 12-17 May 1996, ESA SP389, 1996.
Kivelson, M. G., Khurana, K. K., Walker, R. J., Kepko, L., and Xu, D.: Flux ropes, interhemispheric conjugacy, and magnetospheric current closure, J. Geophys. Res., 101, 27341-27350, 1996.

Lyon, J. G., Fedder, J. A., and Mobarry, C. M.: The Lyon-FedderMobarry (LFM) global MHD magnetospheric simulation code, J. Atmos. Solar-Terr. Phy., 66, 1333-1350, 2004.

McPherron, R. L.: Physical Processes Producing Magnetospheric Substorms and Magnetic Storms, Geomagnetism vol. 4, J. Jacobs, Academic Press Ltd., London, England, 593-739, available at: http://geomag.usgs.gov/iagaxiii/papers/McPherron_ 1991_JacobsBook.pdf, 1991.

Moldwin, M. B. and Hughes, W. J.: On the Formation and Evolution of Plasmoids: A Survey of ISEE 3 Geotail Data, J. Geophys. Res., 97, 19259-19282, 1992.

Moldwin, M. B. and Hughes, W. J.: Geomagnetic Substorm Association of Plasmoids, J. Geophys. Res., 98, 81-88, 1993.

Nagai, T., Nakamura, R., Mukai, T., Yamamoto, T., Nishida, A., and Kokubun, S.: Substorms, tail flows and plasmoids, Adv. Space Res., 20, 961-971, 1997.

Palmroth, M., Pulkkinen, T. I., Janhunen, P., and Wu, C.-C.: Stormtime energy transfer in global MHD simulation, J. Geophys. Res., 108, 1048, doi:10.1029/2002JA009446, 2003.

Palmroth, M., Koskinen, H. E. J., Pulkkinen, T. I., Toivanen, P. K., Janhunen, P., Milan, S. E., and Lester, M.: Magnetospheric feedback in solar wind energy transfer, J. Geophys. Res., 115, A00I10, doi:10.1029/2010JA015746, available at: http://www. agu.org/journals/ja/ja1012/2010JA015746/, 2010.

Pulkkinen, T. I., Baker, D. N., Frank, L. A., Sigwarth, J. B., Opgenoorth, H. J., Greenwald, R., Friis-Christensen, E., Mukai, T., Nakamura, R., Singer, H., Reeves, G. D., and Lester, M.: Two substorm intensifications compared: Onset, expansion, and global consequences, J. Geophys. Res., 103, 15-27, 1998.

Silbergleit, V. M., Zossi de Artigas, M. M., and Manzano J. R.: Energy dissipation in substorms: plasmoids ejection, J. Atmos. Solar-Terr. Phy., 59, 1355-1358, 1997.

Slavin, J. A., Daly, P. W., Smith, E. J., Sanderson, T. R., Wenzel, K.-P., Lepping, R. P., and Kroehl, H. W.: Magnetic configuration of the distant plasma sheet: ISEE 3 observations, Magnetotail Physics, Lui, A. T. Y., The Johns Hopkins University Press, Baltimore, 59-63, 1987.

Slinker, S. P., Fedder, J. A., and Lyon, J. G.: Plasmoid formation and evolution in a numerical simulation of a substorm, Geophys. Res. Lett., 22, 859-862, 1995.

Zong, Q.-G., Fritz, T. A., Pu, Z. Y., Fu, S. Y., Baker, D. N., Zhang, H., Lui, A. T., Vogiatzis, I., Glassmeier, K.-H., Korth, A., Daly, P. W., Balogh, A., and Reme, H.: Cluster observations of earthward flowing plasmoid in the tail, Geophys. Res. Lett., 31, L18803, doi:10.1029/2004GL020692, 2004. 